\title{
The Logistic Inverse Lomax Distribution with Properties and Applications
}

\author{
Ramesh Kumar Joshi ${ }^{1}$, Vijay Kumar ${ }^{2}$ \\ ${ }^{1}$ Associate Professor, Trichandra Multiple Campus, Saraswoti Sadan, Kathmandu, Nepal \\ ${ }^{2}$ Professor, Department of Mathematics and Statistics, DDU Gorakhpur University, Gorakhpur, India
}

\begin{tabular}{|c|c|}
\hline ARTICLE INFO & ABSTRACT \\
\hline Published Online: & In this article, a three-parameter continuous distribution is introduced called Logistic inverse Lomax \\
\hline 20 January 2021 & $\begin{array}{l}\text { distribution. We have discussed some mathematical and statistical properties of the distribution such } \\
\text { as the probability density function, cumulative distribution function and hazard rate function, } \\
\text { survival function, quantile function, the skewness, and kurtosis measures. The model parameters of } \\
\text { the proposed distribution are estimated using three well-known estimation methods namely } \\
\text { maximum likelihood estimation (MLE), least-square estimation (LSE), and Cramer-Von-Mises }\end{array}$ \\
\hline Corresponding Author: & estimation (CVME) methods. The goodness of fit of the proposed distribution is also evaluated by \\
\hline Vijay Kumar & fitting it in comparison with some other existing distributions using a real data set. \\
\hline
\end{tabular}

KEYWORDS: Logistic distribution, Inverse Lomax distribution, Reliability function, MLE.

\section{INTRODUCTION}

Life-time models are generally used to study the length of the life of components of a system, a device, and in general, reliability and survival analysis. Lifetime distributions are frequently used in areas like life science, medicine, biology, engineering, insurance, etc. Many continuous probability distributions such as Cauchy, exponential, gamma, Weibull have been frequently used in statistical literature to analyze lifetime data. For a few years, most of the researchers are attracted towards one parameter logistic distribution for its potential in modeling life-time data, and it has been observed that this distribution has performed excellently in many applications.

The logistic distribution is a univariate continuous distribution and both its PDF and CDF functions have been used in many different areas like logistic regression, logit models and neural networks. The logistic distribution has wider tails than a Gaussian distribution so it is more consistent with the underlying data and provides better insight into the likelihood of extreme events. Let $T$ be a non negative random variable follows the logistic distribution with shape parameter $\delta>0$, and its cumulative distribution function is given by

$$
K(t ; \delta)=\frac{1}{1+e^{-\delta t}} ; \delta>0, t \in \mathfrak{R}
$$

and its corresponding PDF is

$$
k(t ; \delta)=\frac{\delta e^{-\delta t}}{\left(1+e^{-\delta t}\right)^{2}} ; \quad \delta>0, t \in \mathfrak{R}
$$

Tahir et al. (2016) has defined a new generating family of continuous distributions generated from a logistic random variable called the logistic- $X$ family. Its density function can be symmetrical, left-skewed, right-skewed and reversed-J shaped, and can have increasing, decreasing, bathtub and upside-down bathtub hazard rates shaped. Joshi et al. (2020) has introduced logistic exponential power and its hazard function can exhibit increasing, decreasing, bathtub and upside-down bathtub shaped. Mandouh (2018) has introduced Logistic-modified Weibull distribution which is flexible for survival analysis as compared to modified Weibull distribution. Joshi and Kumar (2020) have created half-logistic NHE distribution and studied its various mathematical and statistical properties. Joshi and Kumar (2020) has introduced Lindely exponential power, Chaudhary \& Kumar (2020) have presented the half logistic exponential extension distribution using the parent distribution as exponential extension distribution. Kumar (2010) have presented the Bayesian analysis of exponential extension distribution. Lan and Leemis (2008) has presented an approach to define the logistic compounded model and 
introduced the logistic-exponential survival distribution. This has several useful probabilistic properties for lifetime modeling. Unlike most distributions in the bathtub and upside down bathtub classes, the logistic-exponential distribution exhibit closed-form density, hazard, cumulative hazard, and survival functions. The survival function of the logisticexponential distribution is

$$
I(y ; v)=\frac{1}{1+\left(e^{v y}-1\right)^{\alpha}} ; \quad \alpha>0, v>0, y \geq 0
$$

Where $\alpha$ and $v$ are shape and scale parameters of LE distribution. Applying the similar approach used by (Lan \& Leemis, 2008) we have introduced the new distribution called logistic inverse Lomax distribution. In this study we have taken inverse Lomax distribution as parent distribution. The Lomax distribution can also be called Pareto Type-II distribution and it can be used in many areas like life science, medicine, engineering, and many more.

The distribution was defined by Lomax (1954) having a heavy-tailed shaped distribution. It can also be used in reliability and life testing problems in engineering and in reliability analysis as an alternative distribution (Hassan \& Al-Ghamdi, 2009). The inverse Lomax (IL) distribution is one of important life-time distribution. The inverse Lomax distribution was introduced by (Kleiber, 2004) and used it to get Lorenz ordering relationship among ordered statistics. The IL is related to the family of generalized beta distribution. Kleiber and Kotz (2003) showed that the IL distribution can be used in economics and actuarial sciences. The IL distribution has a lot of applications in stochastic modeling of decreasing failure rate life components, and life testing. The CDF and PDF of twoparameter inverse Lomax can be expressed as

$$
Z(t ; \beta, \chi)=\{1+(\beta / t)\}^{-\chi} ; t \geq 0, \beta>0, \chi>0
$$

$$
z(t ; \beta, \chi)=\frac{\chi}{\beta^{\chi}} t^{\chi-1}\{1+(\beta / t)\}^{-\chi-1} ; t \geq 0, \beta>0, \chi>0
$$

\section{(1.5)}

The main objective of this study is to introduce a more flexible distribution by inserting just one extra parameter to the inverse Lomax distribution to attain a better fit to the lifetime data sets. We have illustrated some distributional properties and its applicability. The remaining parts of the proposed study are organized as follows. In Section 2 we present the Logistic inverse Lomax distribution and its various mathematical and statistical properties. We have employing some estimation methods to estimate the model parameters namely the maximum likelihood estimation (MLE), least-square estimation (LSE) and Cramer-VonMises estimation (CVME) methods. For the maximum likelihood (ML) estimate, we have constructed the asymptotic confidence intervals using the observed information matrix are presented in Section 3. In Section 4, a real data set has been analyzed to explore the applications and capability of the proposed distribution. In this section, we present the estimated value of the parameters and loglikelihood, AIC, BIC, AICC and HQIC criterion for ML, LSE, and CVME also the goodness of fit of the proposed distribution is also evaluated by fitting it in comparison with some other existing distributions using a real data set. Finally, in Section 5 we present some concluding remarks.

\section{THE LOGISTIC INVERSE LOMAX (LIL) DISTRIBUTION}

In this section we have put forward a new distribution called logistic inverse Lomax (LIL) distribution. We have taken the inverse Lomax as baseline distribution defined by (Kleiber \& Kotz, 2003). Let $X$ be a non-negative random variable with a positive shape parameters $\alpha$ and $\lambda$ and a positive scale parameter $\beta$ then CDF of logistic Lomax distribution can be defined as

$$
F(x)=1-\left[1+\left\{\left(1+\frac{\beta}{x}\right)^{\lambda}-1\right\}^{\alpha}\right]^{-1} ; x \geq 0, \alpha>0, \beta>0, \lambda>0 .
$$

The corresponding PDF of LIL distribution is

$$
f(x)=\frac{\alpha \beta \lambda(1+(\beta / x))^{\lambda-1}\left\{(1+(\beta / x))^{\lambda}-1\right\}^{\alpha-1}}{x^{2}\left[1+\left\{(1+(\beta / x))^{\lambda}-1\right\}^{\alpha}\right]^{2}} ; x \geq 0 .
$$

This CDF function is alike to the log logistic CDF function with the second term of the denominator being changed in its base to IL function, and hence we named it logistic inverse Lomax distribution.

\section{Reliability function}

The reliability function of LIL distribution is

$$
\begin{gathered}
R(x)=1-F(x) \\
=\left[1+\left\{(1+\beta / x)^{\lambda}-1\right\}^{\alpha}\right]^{-1} ; x \geq 0,(\alpha, \beta, \lambda)>0
\end{gathered}
$$

\section{Hazard function}

The failure rate function of LIL distribution can be defined

$$
\begin{gathered}
\text { as, } \quad h(x)=\frac{f(x)}{R(x)} \\
=\frac{\alpha \beta \lambda(1+\beta / x)^{\lambda-1}\left\{(1+\beta / x)^{\lambda}-1\right\}^{\alpha-1}}{x^{2}\left[1+\left\{(1+\beta / x)^{\lambda}-1\right\}^{\alpha}\right]} ; x \geq 0,(\alpha, \beta, \lambda)>0
\end{gathered}
$$

In Figure 1, we have displayed the plots of the PDF of LIL distribution for different values of $\alpha, \beta$ and $\lambda$. 


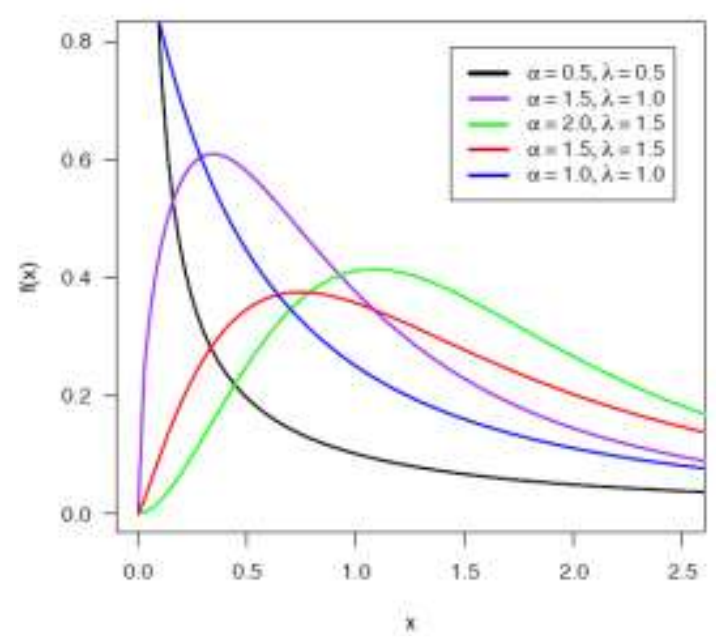

Figure 1. Graph of PDF of LIL distribution

In Figure 2, we have displayed the plots hazard rate function of LIL distribution for different values of $\alpha, \beta$ and $\lambda$.

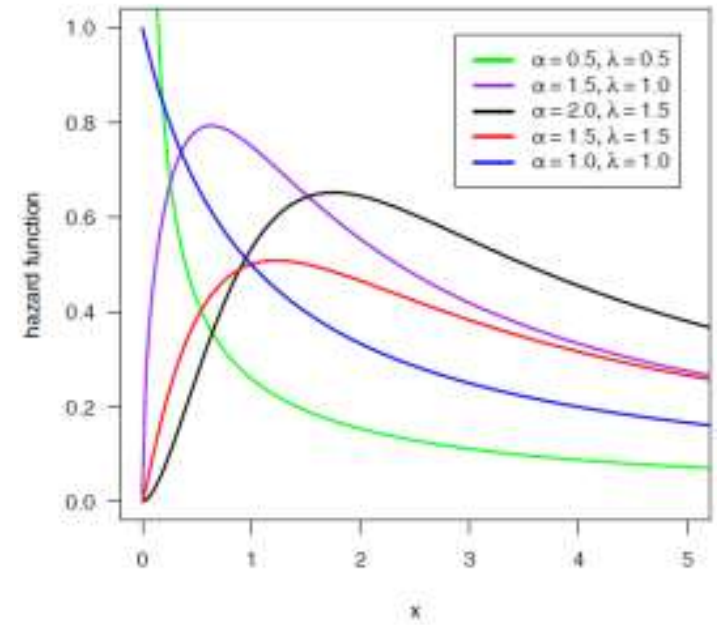

Figure 2. Plots of hazard function for different values of $\alpha$ and $\lambda$.

\section{Quantile function}

The Quantile function of Logistic Lomax distribution can be expressed as

$$
Q(p)=\beta\left[\left\{1+\left(\mathrm{p}^{-1}-1\right)^{1 / \alpha}\right\}^{1 / \lambda}-1\right]^{-1} ; 0<p<1(2.5)
$$

\section{Random deviate generation:}

$x=\beta\left[\left\{1+\left(u^{-1}-1\right)^{1 / \alpha}\right\}^{1 / \lambda}-1\right]^{-1} ; 0<u<1(2.6)$

\section{Skewness and Kurtosis:}

The measures of Skewness based on quantiles is Bowley's coefficient of skewness and it can be expressed as

$$
\text { Skewness }=\frac{Q(0.75)+Q(0.25)-2 Q(0.5)}{Q(0.75)-Q(0.25)} \text { and (2.7) }
$$

Coefficient of kurtosis based on octiles which was defined by (Moors, 1988) is

$$
K_{u}(M)=\frac{Q(0.875)-Q(0.625)+Q(0.375)-Q(0.125)}{Q(3 / 4)-Q(1 / 4)},
$$

\section{METHODS OF ESTIMATION}

In this section, the parameters of the LIL distribution are estimated by using maximum likelihood estimation method. Let, $x_{1}, x_{2}, \ldots, x_{n}$ is a random sample from $\operatorname{LIL}(\alpha, \beta, \lambda)$ and the likelihood function, $L(\alpha, \beta, \lambda)$ is given by,

$$
\begin{aligned}
& L\left(\psi ; x_{1}, x_{2} \ldots x_{n}\right)=f\left(x_{1}, x_{2}, \ldots x_{n} / \psi\right)=\prod_{i=1}^{n} f\left(x_{i} / \psi\right) \\
& L(\alpha, \beta, \lambda)=\alpha \beta \lambda \prod_{i=1}^{n} \frac{\left(1+\beta / x_{i}\right)^{\lambda-1}\left(\left(1+\beta / x_{i}\right)^{\lambda}-1\right)^{\alpha-1}}{\left\{1+\left(\left(1+\beta / x_{i}\right)^{\lambda}-1\right)^{\alpha}\right\}^{2}} ; x \geq 0 . ;(\alpha, \beta, \lambda)>0, x>0
\end{aligned}
$$

Now log-likelihood density is

$$
\begin{aligned}
l= & n \ln \alpha+n \ln \beta+n \ln \lambda-2 \sum_{i=1}^{n} \log x_{i}+(\lambda-1) \sum_{i=1}^{n} \log \left(1+\beta / x_{i}\right) \\
& +(\alpha-1) \sum_{i=1}^{n} \log \left\{\left(1+\beta / x_{i}\right)^{\lambda}-1\right\}-2 \sum_{i=1}^{n} \log \left[1+\left\{\left(1+\beta / x_{i}\right)^{\lambda}-1\right\}^{\alpha}\right]
\end{aligned}
$$

Differentiating (3.1) with respect to $\alpha, \beta$ and $\lambda$ we get,

$$
\begin{aligned}
\frac{\partial l}{\partial \alpha}= & \frac{n}{\alpha}+\sum_{i=1}^{n} \log \left\{\left(1+\beta / x_{i}\right)^{\lambda}-1\right\}-2 \sum_{i=1}^{n} \frac{\left[\left(1+\beta / x_{i}\right)^{\lambda}-1\right]^{\alpha} \log \left\{\left(1+\beta / x_{i}\right)^{\lambda}-1\right\}}{1+\left\{\left(1+\beta / x_{i}\right)^{\lambda}-1\right\}^{\alpha}} \\
\frac{\partial l}{\partial \beta}= & \frac{n}{\beta}+(\lambda-1) \sum_{i=1}^{n} \frac{1}{x_{i}\left(1+\beta / x_{i}\right)}+\lambda(\alpha-1) \sum_{i=1}^{n} \frac{\left(1+\beta / x_{i}\right)^{\lambda-1}}{\left\{\left(1+\beta / x_{i}\right)^{\lambda}-1\right\} x_{i}} \\
& -2 \alpha \lambda \sum_{i=1}^{n} \frac{\left\{\left(1+\beta / x_{i}\right)^{\lambda}-1\right\}^{\alpha-1}\left(1+\beta / x_{i}\right)^{\lambda-1}}{x_{i}\left[1+\left\{\left(1+\beta / x_{i}\right)^{\lambda}-1\right\}^{\alpha}\right]} \\
\frac{\partial l}{\partial \lambda}= & \frac{n}{\lambda}+\sum_{i=1}^{n} \log \left(1+\beta / x_{i}\right)+(\alpha-1) \sum_{i=1}^{n} \frac{\left(1+\beta / x_{i}\right)^{\lambda} \log \left(1+\beta / x_{i}\right)}{\log \left(1+\beta / x_{i}\right)^{\lambda}-1} \\
& -2 \alpha \sum_{i=1}^{n} \frac{\left\{\left(1+\beta / x_{i}\right)^{\lambda}-1\right\}^{\alpha-1}\left(1+\beta / x_{i}\right)^{\lambda} \log \left(1+\beta / x_{i}\right)}{1+\left\{\left(1+\beta / x_{i}\right)^{\lambda}-1\right\}^{\alpha}}
\end{aligned}
$$

Equating above three nonlinear equations to zero and solving simultaneously for $\alpha, \beta$ and $\lambda$, we get the maximum likelihood estimate $\hat{\alpha}, \hat{\beta}$ and $\hat{\lambda}$ of the parameters $\alpha, \beta$ and $\lambda$. By using computer software like R, Matlab, Mathematica etc for maximization of (3.1) we can obtain the estimated value of $\alpha$, $\beta$ and $\lambda$. For the confidence interval estimation of $\alpha, \beta$ and $\lambda$ and testing of the hypothesis, we have to calculate the observed information matrix. The observed information matrix for $\alpha, \beta$ and $\lambda$ can be obtained as,

$$
U=\left[\begin{array}{lll}
U_{11} & U_{12} & U_{13} \\
U_{21} & U_{22} & U_{23} \\
U_{31} & U_{32} & U_{33}
\end{array}\right]
$$

where

$$
\begin{gathered}
U_{11}=\frac{\partial^{2} l}{\partial \alpha^{2}}, U_{12}=\frac{\partial^{2} l}{\partial \alpha \partial \beta}, U_{13}=\frac{\partial^{2} l}{\partial \alpha \lambda} \\
U_{21}=\frac{\partial^{2} l}{\partial \beta \partial \alpha}, U_{22}=\frac{\partial^{2} l}{\partial \beta^{2}}, U_{23}=\frac{\partial^{2} l}{\partial \beta \partial \lambda}
\end{gathered}
$$




$$
U_{31}=\frac{\partial^{2} l}{\partial \lambda \partial \alpha}, U_{32}=\frac{\partial^{2} l}{\partial \beta \partial \lambda}, U_{33}=\frac{\partial^{2} l}{\partial \lambda^{2}}
$$

Let $\Omega=(\alpha, \beta, \lambda)$ denote the parameter space and the corresponding MLE of $\Omega$ as $\hat{\Omega}=(\hat{\alpha}, \hat{\beta}, \hat{\lambda})$, then $(\hat{\Omega}-\Omega) \rightarrow N_{3}\left[0,(U(\Omega))^{-1}\right]$ where $U(\Omega)$ is the

Fisher's information matrix. Using the Newton-Raphson algorithm to maximize the likelihood creates the observed information matrix and hence the variance-covariance matrix is obtained as,

$$
[U(\Omega)]^{-1}=\left(\begin{array}{ccc}
\operatorname{var}(\hat{\alpha}) & \operatorname{cov}(\hat{\alpha}, \hat{\beta}) & \operatorname{cov}(\hat{\alpha}, \hat{\lambda}) \\
\operatorname{cov}(\hat{\alpha}, \hat{\beta}) & \operatorname{var}(\hat{\beta}) & \operatorname{cov}(\hat{\beta}, \hat{\lambda}) \\
\operatorname{cov}(\hat{\alpha}, \hat{\lambda}) & \operatorname{cov}(\hat{\beta}, \hat{\lambda}) & \operatorname{var}(\hat{\lambda})
\end{array}\right)
$$

Hence from the asymptotic normality of MLEs, approximate $100(1-\alpha) \%$ confidence intervals for $\alpha, \beta$ and $\lambda$ can be constructed as,

$$
\begin{gathered}
\hat{\alpha} \pm Z_{\alpha / 2} S E(\hat{\alpha}), \hat{\beta} \pm Z_{\alpha / 2} S E(\hat{\beta}) \text { and } \\
\hat{\lambda} \pm Z_{\alpha / 2} S E(\hat{\lambda})
\end{gathered}
$$

where $Z_{\alpha / 2}$ is the upper percentile of standard normal variate

\section{APPLICATIONS TO REAL DATASET}

In this section, we illustrate the applicability of logistic inverse Lomax distribution using two real datasets used by earlier researchers.

\section{Dataset-I}

This is a real data set represents the remission times (in months) of a random sample of 128 bladder cancer patients (Lee \& Wang, 2003): sorted data

$0.08,0.20,0.40,0.50,0.51,0.81,0.90,1.05,1.19,1.26,1.35$, $1.40,1.46,1.76,2.02,2.02,2.07,2.09,2.23,2.26,2.46,2.54$, $2.62,2.64,2.69,2.69,2.75,2.83,2.87,3.02,3.25,3.31,3.36$, $3.36,3.48,3.52,3.57,3.64,3.70,3.82,3.88,4.18,4.23,4.26$, $4.33,4.34,4.40,4.50,4.51,4.87,4.98,5.06,5.09,5.17,5.32$, 5.32, 5.34, 5.41, 5.41, 5.49, 5.62, 5.71, 5.85, 6.25, 6.54, 6.76, $6.93,6.94,6.97,7.09,7.26,7.28,7.32,7.39,7.59,7.62,7.63$, $7.66,7.87,7.93,8.26,8.37,8.53,8.65,8.66,9.02,9.22,9.47$, $9.74,10.06,10.34,10.66,10.75,11.25,11.64,11.79,11.98$, $12.02,12.03,12.07,12.63,13.11,13.29,13.80,14.24,14.76$, 14.77, 14.83, 15.96, 16.62, 17.12, 17.14, 17.36, 18.10, 19.13, $20.28,21.73,22.69,23.63,25.74,25.82,26.31,32.15,34.26$, $36.66,43.01,46.12,79.05$

The MLEs are calculated by utilizing the optim() function in $\mathrm{R}$ software (R Core Team, 2020) and (Mailund, 2017) by maximizing the likelihood function (3.1). We have obtained Log-Likelihood value is $l=-409.4909$ and the MLE's with their standard errors (SE) and 95\% confidence interval for $\alpha$, $\beta$, and $\lambda$ are presented in Table 1 .
Table 1. MLE and SE and $95 \%$ confidence interval for $\alpha, \beta$ and $\lambda$

\begin{tabular}{llll}
\hline \multicolumn{2}{l}{ Parameter MLE } & SE & 95\% ACI \\
\hline alpha & 2.87951 & 0.25522 & $(2.3793,3.3797)$ \\
beta & 38.51405 & 6.56328 & $(25.6500,51.3781)$ \\
lambda & 0.35313 & 0.02929 & $(0.2957,0.4105)$ \\
\hline
\end{tabular}

We have displayed the graph of the profile log-likelihood function of $\alpha, \beta$, and $\lambda$ in Figure 3 (Kumar \& Ligges, 2011) and observed that the MLEs are unique.
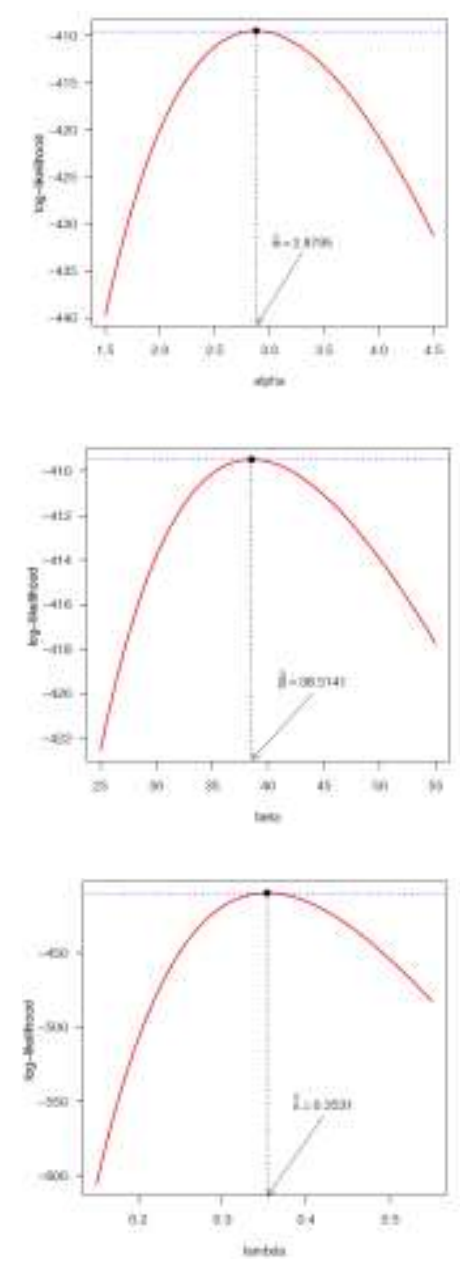

Figure 3. Graph of profile log-likelihood function of $\alpha, \beta$, and $\lambda$.

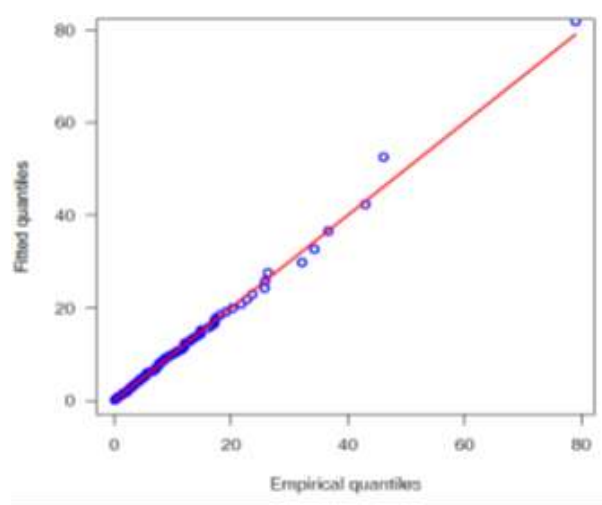




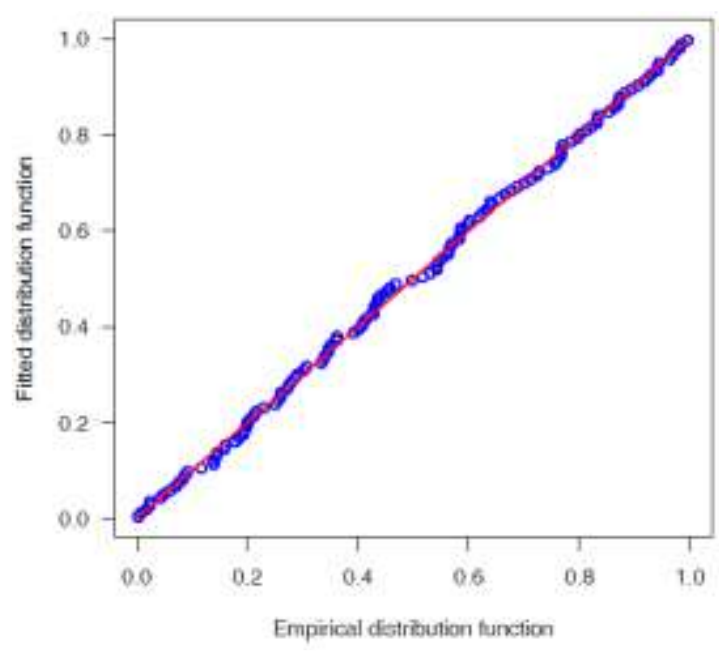

Figure 4. The Q-Q plot (left panel) and P-P plot (right panel) of LIL distribution

\section{Dataset-II}

The data given below represents the fatigue life of 6061-T6 aluminum coupons cut parallel to the direction of rolling and oscillated at 18 cycles per seconds (cps) which consists of 101 observations with maximum stress per cycle 31,000 psi. This data set was originally analyzed by (Birnbaum \& Saunders, 1969).

70, 90, 96, 97, 99, 100, 103, 104, 104, 105, 107, 108, $108,108,109,109,112,112,113,114,114,114,116,119$, $120,120,120,121,121,123,124,124,124,124,124,128$, $128,129,129,130,130,130,131,131,131,131,131,132$, $132,132,133,134,134,134,134,134,136,136,137,138$, $138,138,139,139,141,141,142,142,142,142,142,142$, $144,144,145,146,148,148,149,151,151,152,155,156$, $157,157,157,157,158,159,162,163,163,164,166,166$, $168,170,174,196,212$

We have obtained Log-Likelihood value is $l=-455.9934$ and the MLE's with their standard errors (SE) and 95\% confidence interval for $\alpha, \beta$, and $\lambda$ are presented in Table 2 .

Table 2. MLE and SE and 95\% confidence interval for $\alpha, \beta$ and $\lambda$

\begin{tabular}{llll}
\hline \multicolumn{2}{l}{ Parameter MLE } & SE & 95\% ACI \\
\hline alpha & 9.0749 & 0.7225 & $(7.6588,10.491)$ \\
beta & 55.5687 & 3.6024 & $(48.508,62.6294)$ \\
lambda & 1.9797 & 0.1114 & $(1.7614,2.1980)$ \\
\hline
\end{tabular}

We have displayed the graph of the profile log-likelihood function of $\alpha, \beta$, and $\lambda$ in Fig. 4 (Kumar \& Ligges, 2011) and observed that the MLEs are unique.
In Figure 5 we have presented the Q-Q plot (empirical quantile against theoretical quantile) and P-P plot of LIL distribution.
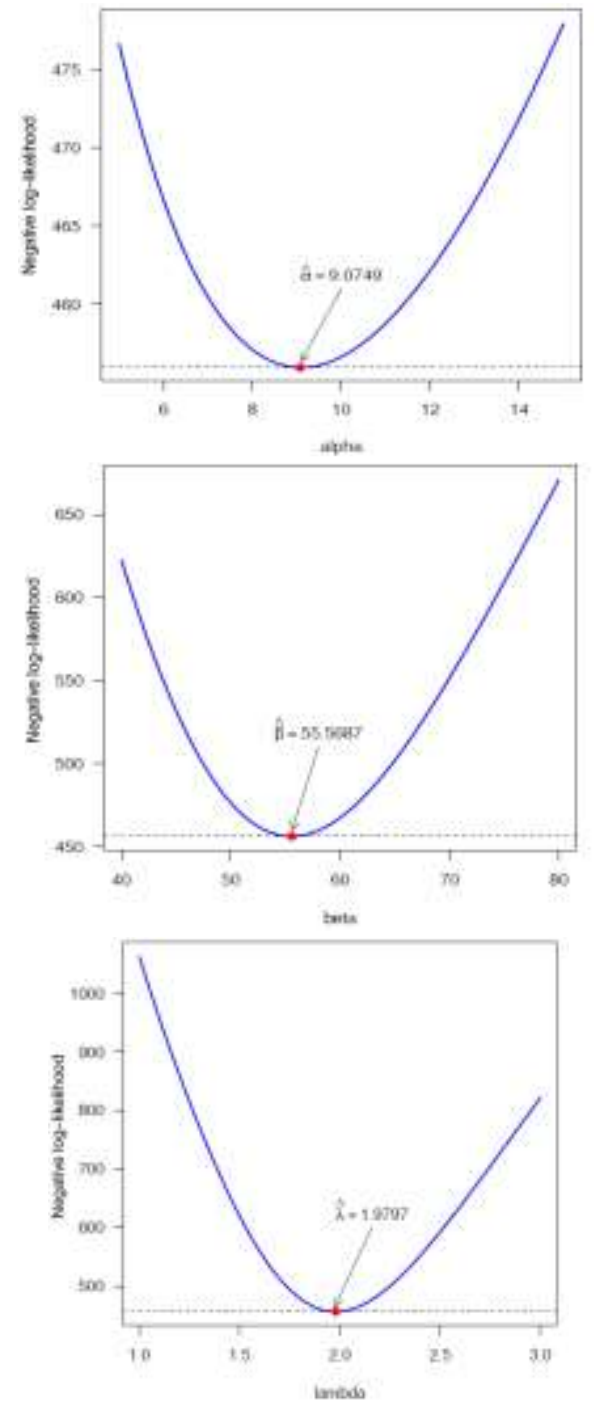

Figure 5. Graph of profile log-likelihood function of $\alpha, \beta$, and $\lambda$.

In Figure 6 we have presented the Q-Q plot (empirical quantile against theoretical quantile) and P-P plot of LIL distribution.

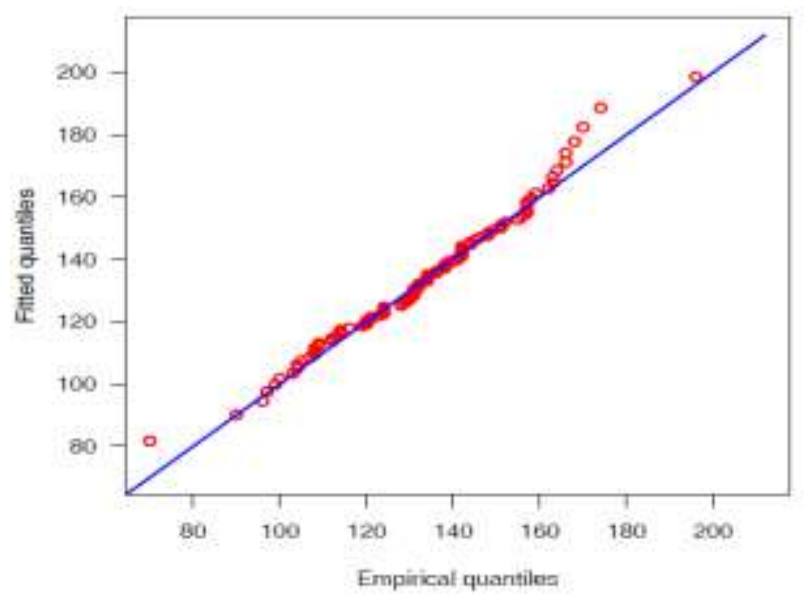




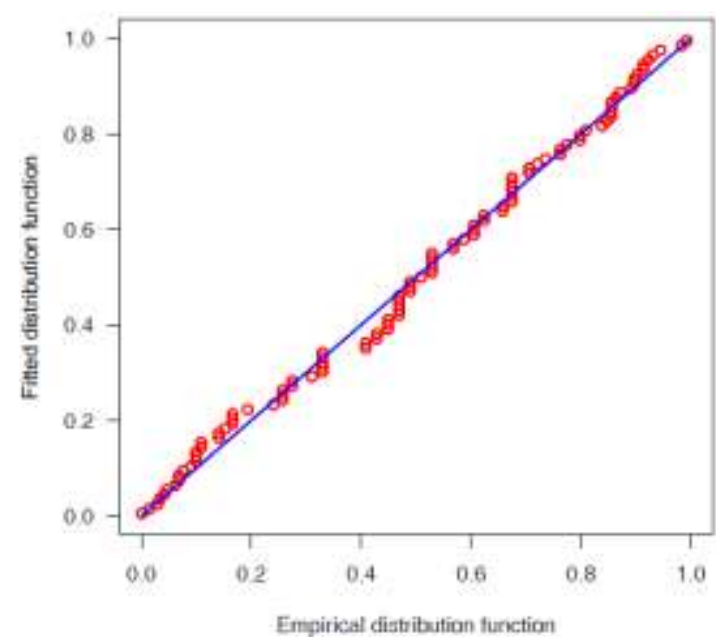

Figure 6. The Q-Q plot (left panel) and P-P plot (right panel) of LIL distribution

To illustrate the goodness of fit of the LIL distribution, we have select some well-known distribution for comparison purpose which are listed blew,

\section{A. Inverted Lomax (IL) distribution:}

The cumulative probability distribution function of IL introduced by (Lan \& Leemis, 2008) having upside down bathtub-shaped hazard function distribution with parameters $\begin{array}{llll}\beta & \text { and } & \lambda\end{array}$ $F_{I L}(x)=\{1+(\beta / x)\}^{-\lambda} ; x \geq 0, \beta>0, \lambda>0$.

B. Exponentiated Lomax (EL) distribution: The CDF of exponentiated lomax introduced by (Lan \& Leemis, 2008) can be written as

Table 3. Log-likelihood (LL), AIC, BIC, CAIC and HQIC (dataset-I)

\begin{tabular}{llllll}
\hline Distribution & -LL & AIC & BIC & CAIC & HQIC \\
\hline LIL & 409.4909 & 824.9818 & 833.5379 & 825.1753 & 828.4582 \\
GEE & 410.6013 & 827.2026 & 835.7586 & 827.3961 & 830.6789 \\
IEL & 409.9542 & 825.9084 & 834.4645 & 826.1020 & 829.3848 \\
EL & 410.0718 & 826.1436 & 834.6997 & 826.3372 & 829.6200 \\
GE & 413.0776 & 830.1552 & 835.8592 & 830.2512 & 832.4728 \\
EP & 426.6474 & 857.2948 & 862.9989 & 857.3893 & 859.6124 \\
\hline
\end{tabular}

Table 4. Log-likelihood (LL), AIC, BIC, CAIC and HQIC (dataset-II)

\begin{tabular}{llllll}
\hline Distribution & -LL & AIC & BIC & CAIC & HQIC \\
\hline LIL & 455.9934 & 917.9869 & 925.8322 & 918.2343 & 921.1629 \\
GEE & 456.4468 & 918.8935 & 926.7389 & 919.1409 & 922.0696 \\
IEL & 457.0925 & 920.1850 & 928.0304 & 920.4324 & 923.3610 \\
EL & 462.9163 & 931.8327 & 939.6780 & 932.0801 & 935.0087 \\
GE & 463.7324 & 931.4648 & 936.6951 & 931.5873 & 933.5822 \\
EP & 476.7897 & 957.5794 & 962.8096 & 957.6994 & 959.6967 \\
\hline
\end{tabular}


For the both datasets we have presented the Histogram and the density function of fitted distributions and Empirical distribution function with the estimated distribution function

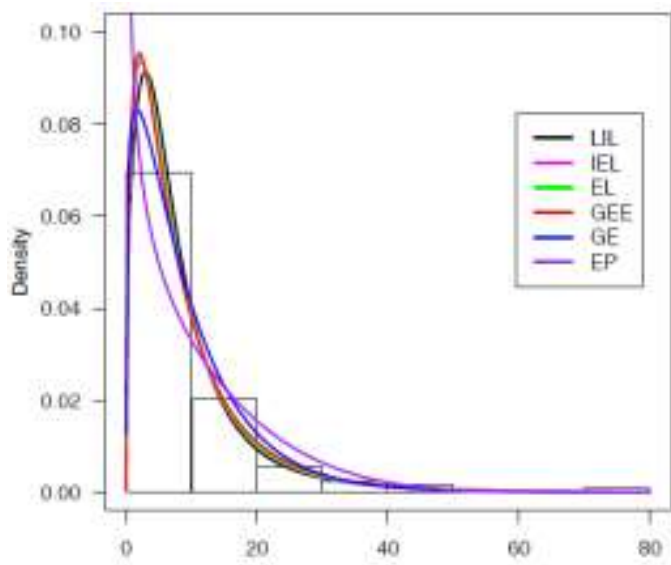

$\times$

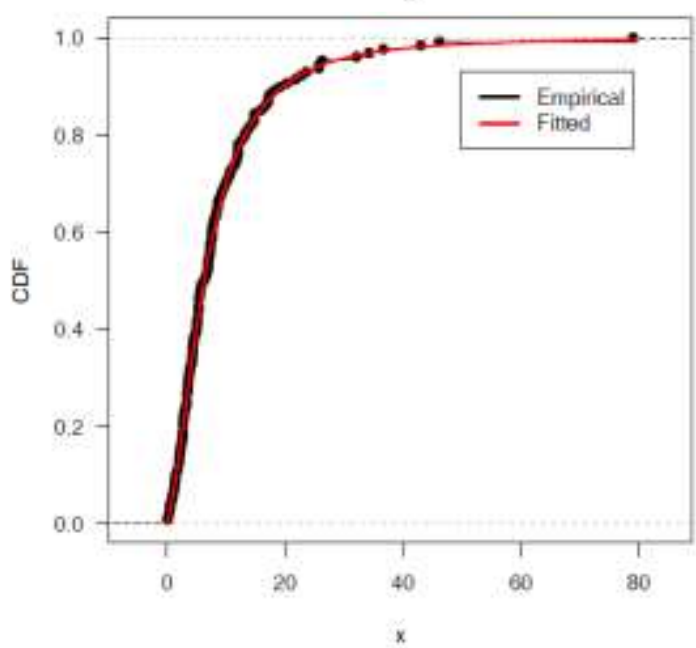

of LIL and some selected distributions are presented in Figure 7.
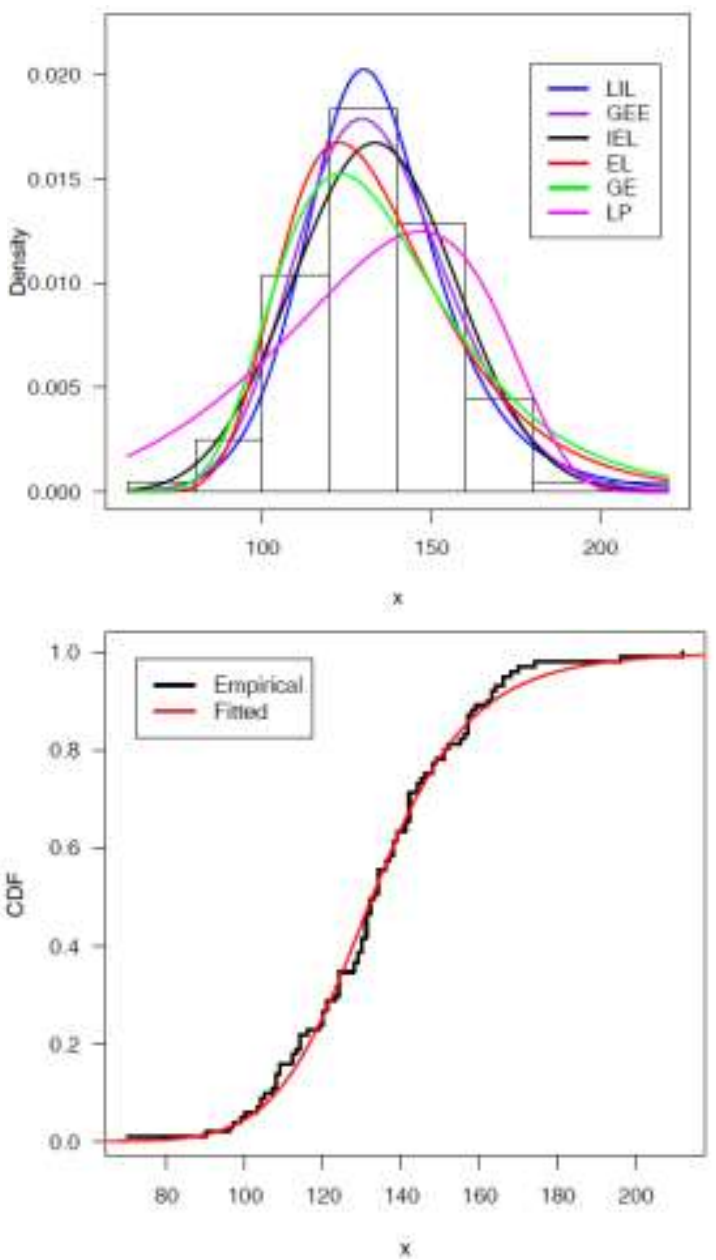

Figure 7. The Histogram and the density function of fitted distributions for data-I and data-II (first row) and Empirical distribution function with estimated distribution function for data-I and data-II (second row)

To compare the goodness-of-fit of the LIL distribution with other competing distributions we have presented the value of Kolmogorov-Simnorov (KS), the Anderson-Darling (W) and the Cramer-Von Mises $\left(\mathrm{A}^{2}\right)$ statistics in Table 5 and Table 6.
It is observed that the LIL distribution has the minimum value of the test statistic and higher $p$-value thus we conclude that the LIL distribution gets quite better fit and more consistent and reliable results from others taken for comparison.

Table 5. The goodness-of-fit statistics and their corresponding p-value (dataset-I)

\begin{tabular}{lccc}
\hline Distribution & KS $(\boldsymbol{p}$-value $)$ & $\boldsymbol{W}(\boldsymbol{p}$-value $)$ & $\boldsymbol{A}^{2}(\boldsymbol{p}$-value $)$ \\
\hline LIL & $0.0297(0.9998)$ & $0.0137(0.9998)$ & $0.0898(0.9999)$ \\
GEE & $0.0442(0.9636)$ & $0.0394(0.9367)$ & $0.2630(0.9631)$ \\
IEL & $0.03888(0.9903)$ & $0.0233(0.9929)$ & $0.1607(0.9977)$ \\
EL & $0.0405(0.9846)$ & $0.0262(0.9871)$ & $0.1798(0.9950)$ \\
GE & $0.0725(0.5115)$ & $0.1279(0.4652)$ & $0.7137(0.5472)$ \\
EP & $0.1199(0.0503)$ & $0.5993(0.0223)$ & $3.6745(0.0126)$ \\
\hline
\end{tabular}

Table 6. The goodness-of-fit statistics and their corresponding p-value (dataset-II)

\begin{tabular}{cccc}
\hline Distribution & $\boldsymbol{K} \boldsymbol{S}(\boldsymbol{p}$-value $)$ & $\boldsymbol{W}(\boldsymbol{p}$-value $)$ & $\boldsymbol{A}^{2}(\boldsymbol{p}$-value $)$ \\
\hline LIL & $0.0626(0.8241)$ & $0.0527(0.8606)$ & $0.3776(0.8703)$ \\
GEE & $0.0755(0.6132)$ & $0.0682(0.7640)$ & $0.4132(0.8352)$ \\
IEL & $0.0690(0.7224)$ & $0.0858(0.6601)$ & $0.5809(0.6658)$ \\
\hline
\end{tabular}


"The Logistic Inverse Lomax Distribution with Properties and Applications"

\begin{tabular}{lccc}
\hline EL & $0.1077(0.1916)$ & $0.2341(0.2102)$ & $1.4750(0.1826)$ \\
GE & $0.1066(0.2014)$ & $0.3112(0.1257)$ & $2.0724(0.0840)$ \\
EP & $0.1378(0.0433)$ & $0.6942(0.0130)$ & $4.5057(0.0050)$ \\
\hline
\end{tabular}

\section{CONCLUSIONS}

In this article, we have generated a three-parameter continuous distribution named Logistic inverse Lomax distribution. Some mathematical and statistical properties of the proposed distribution are presented such as the shapes of the probability density, cumulative density and hazard rate functions, survival function, quantile function, the skewness, and kurtosis measures are derived and established and found that the proposed model is flexible and increasing and $\mathrm{j}$ shaped shaped hazard function. The model parameters are estimated by using maximum likelihood estimation (MLE) method. Two real datasets is considered to explore the applicability and suitability of the proposed distribution and found that the proposed model is quite better than other lifetime model taken into consideration. We hope this model may be an alternative in the field of survival analysis, probability theory and applied statistics.

\section{REFERENCES}

1. Birnbaum, Z.W., \& Saunders, S.C. (1969). Estimation for a family of life distributions with applications to fatigue, Journal of Applied Probability, 6, 328 -347.

2. Chaudhary, A. K. \& Kumar, V. (2020). Half logistic exponential extension distribution with Properties and Applications. International Journal of Recent Technology and Engineering (IJRTE), 8(3), 506512.

3. Chaudhary, A. K. \& Kumar, V. (2020). Lindley half Cauchy distribution: Properties and Applications. International Journal for Research in Applied Science \& Engineering Technology (IJRASET), 8(9), 1233-1242.

4. Gupta, R. D., \& Kundu, D. (2007). Generalized exponential distribution: Existing results and some recent developments. Journal of Statistical Planning and Inference, 137(11), 3537-3547.

5. Hassan, A. and Al-Ghamdi, A. (2009). Optimum step stress accelerated life testing for Lomax distribution. Journal of Applied Sciences Research, 5, 2153-2164.

6. Joshi, R. K. \& Kumar, V. (2020). Lindley exponential power distribution with Properties and Applications. International Journal for Research in Applied Science \& Engineering Technology (IJRASET), 8(10), 22-30.

7. Joshi, R. K. \& Kumar, V. (2020). Half Logistic NHE: Properties and Application. International
Journal for Research in Applied Science \& Engineering Technology (IJRASET), 8(9), 742-753.

8. Joshi, R. K., Sapkota, L.P. \& Kumar, V. (2020). The Logistic-Exponential Power Distribution with Statistical Properties and Applications, International Journal of Emerging Technologies and Innovative Research, 7(12), 629-641

9. Kleiber, C. (2004). Lorenz ordering of order statistics from log-Logistic and related distributions. Journal of Statistical Planning and Inference, 120, 13-19.

10. Kleiber, C., \& Kotz, S. (2003). Statistical size distributions in economics and actuarial sciences. John Wiley and Sons, Inc., Hoboken, New Jersey.

11. Kumar, V. (2010). Bayesian analysis of exponential extension model. J. Nat. Acad. Math, 24, 109-128.

12. Kumar, V. and Ligges, U. (2011). reliaR: A package for some probability distributions, http://cran.rproject.org/web/packages/reliaR/index.html.

13. Lan, Y., \& Leemis, L. M. (2008). The logisticexponential survival distribution. Naval Research Logistics (NRL), 55(3), 252-264.

14. Lee, E. T. \& Wang, J. (2003). Statistical methods for survival data analysis (Vol. 476). John Wiley \& Sons.

15. Lemonte, A. J. (2013). A new exponential-type distribution with constant, decreasing, increasing, upside-down bathtub and bathtub-shaped failure rate function. Computational Statistics \& Data Analysis, 62, 149-170.

16. Lomax, K. S. (1954). Business failures: Another example of the analysis of failure data. Journal of the American Statistical Association, 49(268), 847852.

17. Mailund, T. (2017). Functional Programming in R: Advanced Statistical Programming for Data Science, Analysis and Finance. Apress, Aarhus N, Denmark ISBN-13 (pbk): 978-1-4842-2745-9 ISBN-13 (electronic): 978-1-4842-2746-6 DOI 10.1007/978-1-4842-2746-6

18. Mandouh, R. M. (2018). Logistic-modified weibull distribution and parameter estimation. International Journal of Contemporary Mathematical Sciences, 13(1), 11-23.

19. Moors, J. (1988). A quantile alternative for kurtosis. The Statistician, 37, 25-32.

20. R Core Team (2020). R: A language and environme nt for statistical computing. R Foundation for Statis tical Computing, Vienna, Austria. URL https://www .R-project.org/. 
"The Logistic Inverse Lomax Distribution with Properties and Applications"

21. Smith, R.M. and Bain, L.J. (1975). An exponential power life-test distribution, Communications in Statistics, 4, 469-481

22. Tahir, M. H., Cordeiro, G. M., Alzaatreh, A., Mansoor, M., \& Zubair, M. (2016). The logistic-X family of distributions and its applications. Communications in Statistics-Theory and Methods, 45(24), 7326-7349. 